\title{
Hapten-specific T-Cell Unresponsiveness Induced by Benzylpenicilloyl Autologous Gamma Globulin Conjugates in Human Lymphocytes In Vitro
}

\author{
Raif S. Geha, Lazar Fruchter, and Yves Borel, \\ Divisions of Allergy and Immunology, Department of Medicine, \\ Children's Hospital Medical Center and the Department of Pediatrics, \\ Harvard Medical School, Boston, Massachusetts (0211.5
}

\begin{abstract}
A в S T RACT The aim of these studies was to determine whether unresponsiveness to the main determinant of penicillin, benzylpenicilloyl, can be induced in human peripheral lymphocytes in vitro by conjugates of benzylpenicilloyl (BPO) autologous gamma globulin (HGG). Initially it was shown that conjugates of BPO-keyhole limpet hemocyanin (KLH) elicited lymphocyte proliferation in the peripheral blood lymphocytes of six out of nine adult individuals in vitro. In contrast, conjugates of dinitrophenylated KLH and of BPO-HGG and the carriers HGG and KLH alone failed to do so. Similarly, release of the nonspecific helper factor, lymphocyte mitogenic factor (LMF) occurred only after BPO-KLH stimulation. LMF activity was measured by B-cell proliferation and incorporation of radioactive amino acids into secreted immunoglobulin.
\end{abstract}

Treatment with BPO-HGG for $24 \mathrm{~h}$ in vitro inhibited BPO-KLH-induced lymphocyte proliferation and LMF release. Treatment with either HGG, dinitrophenylated HGG, BPO-KLH, or BPO-human serum albumin failed to abrogate $\mathrm{T}$-cell lymphocyte proliferation of human lymphocytes in vitro. The antigen specificity of the reduced immunologic responsiveness was further demonstrated by the observation that lymphocytes treated with BPO-HGG for $24 \mathrm{~h}$ in vitro responded normally to tetanus toxoid antigen.

The data suggest that conjugates of BPO-HGG induce hapten-specific helper T-cell unresponsiveness in vitro.

\section{INTRODUCTION}

Induction of immunologic tolerance by haptens coupled to nonimmunogenic carriers has been useful in understanding the cellular basis of self vs. nonself

Received for publication 5 April 1.979 and in revised form 4 February 1980. discrimination and in applying tolerance therapeutically in experimental autoimmune disease (1-3). One area of potential therapeutic application of immunologic tolerance is allergy. For example, it has been shown that immunoglobulin $(\mathrm{Ig}) \mathrm{E}$ antibody to the major determinant of penicillin can be suppressed in primed animals by the administration of benzylpenicilloyl conjugated to either isologous IgG or to a synthetic carrier such as D-glutamic acid and D-lysine (4-7). Before applying the concept of carrier-induced tolerance to problems in clinical allergy, it is critical to demonstrate that unresponsiveness can be induced in human lymphocytes in vitro. The major determinant of penicillin, benzylpenicilloyl (BPO) ${ }^{1}$ was selected because of its relevance to immediate hypersensitivity in man $(8-10)$. Here we show that BPO conjugated to autologous human gamma globulin (HGG) specifically suppressed lymphocyte proliferation and the release of lymphocyte mitogenic factor (LMF), a nonspecific T-cell-derived helper factor which induces proliferation and immunoglobulin synthesis in B cells.

\section{METHODS}

\section{Reagents}

HGG was isolated from the serum of nine volunteers over DEAE columns using a $0.01-\mathrm{M}$ pH 6.8 phosphate buffer as

${ }^{1}$ Abbreviations used in this paper: BPO, benzylpenicilloyl; BPO-HGG, benzylpenicilloyl-autologous human gamma globulin; BPO-HSA, benzylpenicilloyl-human serum albumin; BPO-KLH, benzylpenicilloyl-keyhole limpet hemocyanin; DNP, dinitrophenyl; DNP-HGG, dinitrophenylatedautologous human gamma globulin; DNP-KLH, dinitrophenylated keyhole limpet hemocyanin; HGG, human autologous gamma globulin; HSA, human serum albumin; KLH, keyhole limpet hemocyanin; LMF, lymphocyte mitogenic factor; MI, mitogenic indices; $P$, preincubated; PBL, human peripheral blood lymphocyte; $R$, reconstituted; SI, stimulation indices. 
eluting buffer. Keyhole limpet hemocyanin (KLH) was purchased from Sigma Chemical Co., St. Louis, Mo. Human serum albumin (HSA) was obtained as a $25 \%$ solution from Hyland Diagnostics Div., Travenol Laboratories, Inc., Costa Mesa, Calif. BPO was covalently bound in the penicilloyl configuration to protein carriers (KLH, HSA, or HGG) as previously described (11), and residual unbound hapten was removed by gel filtration. The following hapten carrier conjugates were used: $\mathrm{BPO}_{23}-\mathrm{HSA}, \mathrm{BPO}_{56}-\mathrm{KLH}$, and nine preparations of BPO-HGG $\mathrm{BPO}_{4-11}$-HGG; in all cases the suffix numbers refer to the average molar ratio of hapten to carrier (assuming a mol wt of 800,000 for KLH). Dinitrophenyl (DNP) was covalently bound to KLH and HGG as previously described (12). The following hapten carrier conjugates were used: $\mathrm{DNP}_{65}-\mathrm{KLH}$ and three preparations of DNP-HGG, $\mathrm{DNP}_{22-35^{-}}$ HGG. All preparations of hapten carrier protein conjugates were used at the concentration of $100 \mu \mathrm{g} / \mathrm{ml}$. Tetanus toxoid (TT-LP 430 PM, Massachusetts Biological Laboratories) was used as a control antigen at $10 \mu \mathrm{g} / \mathrm{ml}$.

\section{Lymphocyte preparation}

Human peripheral blood lymphocyte (PBL) suspensions were prepared by Ficoll-Hypaque sedimentation of heparinized blood (13). Blood was obtained from nine adult volunteers, all of whom had previously received multiple courses of intramuscular and oral penicillin; none had a positive history of penicillin allergy. All of the donors had also received immunization with TT antigen. Cord blood of six newborns whose mothers did not receive penicillin during pregnancy was also studied.

\section{Culture conditions and assay of DNA synthesis}

Cell suspensions were cultured at a final concentration of $1 \times 10^{6}$ cells $/ \mathrm{ml}$ in complete medium consisting of medium RPMI 1640 containing $10 \% \mathrm{AB}^{+}$serum and $50 \mu \mathrm{g} / \mathrm{ml}$ gentamicin. $0.2-\mathrm{ml}$ aliquots of cell suspensions were distributed into the wells of sterile flat-bottomed microtiter plates (Linbro Chemical Company, Hamden, Conn.). Cultures were made in triplicate, incubated at $37^{\circ} \mathrm{C}$ in a humidified atmosphere of $5 \% \mathrm{CO}_{2}$ in air for $6 \mathrm{~d}$, pulsed with $\left[{ }^{3} \mathrm{H}\right]$ thymidine (New England Nuclear, Boston, Mass., $20 \mathrm{Ci} / \mathrm{mol}, 1 \mu \mathrm{Ci} /$ culture) and harvested $16 \mathrm{~h}$ later as previously described (14). Results were expressed as stimulation indices (SI) where: SI $=$ (counts per minute incorporated by antigen-stimulated culture)/(counts per minute incorporated by control-unstimulated culture).

\section{Production of T-cell helper factors}

Production of T-cell helper factor was assayed as previously described (15). PBL were cultured at $10^{7}$ cells $/ \mathrm{ml}$ for $48 \mathrm{~h}$. One set of cultures received antigen at the onset of the incubation period and were designated $P$ (for preincubated). A second set of cultures received an equivalent amount of antigen at the conclusion of the incubation period and were designated $\mathrm{R}$ (for reconstituted). At the termination of the incubation period, supernates were collected, filtered through a $0.22-\mu \mathrm{m}$ filter (Millipore Corp., Bedford, Mass.) and stored at $-20^{\circ} \mathrm{C}$ until tested.

\section{Assay of T-cell helper factor on B cells}

Paired $P$ and $R$ supernates were assayed for their ability to induce proliferation and immunoglobulin secretion in normal human B cells. B-cell-rich preparations (containing
85-90\% B cells as determined by erythrocyte antibody C3 rosette formation and surface immunofluorescent staining) were obtained from normal human tonsils by fractionation over gradients of bovine serum albumin (15).

DNA synthesis. Supernates were added to B-cell suspensions at a final dilution of 1:2. DNA synthesis in B-cell cultures was measured as described elsewhere (15). Results were expressed as mitogenic indices (MI) where: MI $=\left(\right.$ counts per minute of $\left[{ }^{3} \mathrm{H}\right]$ thymidine incorporated in $\mathrm{P}$ stimulated cultures)/(counts per minute $\left[{ }^{3} \mathrm{H}\right]$ thymidine incorporated in $\mathrm{R}$ stimulated cultures).

\section{Preincubation of lymphocytes with $\mathrm{BPO}$ conjugates and antigens}

Lymphocytes were suspended at a concentration of 1 $\times 10^{7}$ cells $/ \mathrm{ml}$ in complete culture medium in the presence of various BPO or DNP conjugates (100 $\mu \mathrm{g}$ of carrier protein/ $\mathrm{ml})$, of HGG $(100 \mu \mathrm{g} / \mathrm{ml})$, or of TT $(10 \mu \mathrm{g} / \mathrm{ml})$. After a $24-\mathrm{h}$ incubation period the cells were pelleted $(200 \mathrm{~g}$ for $10 \mathrm{~min})$, washed three times in Hanks' balanced salt medium, and then resuspended in complete medium at a final concentration of $1 \times 10^{6}$ cells $/ \mathrm{ml}$. The cells were then incubated $w$ ith various conjugates or with TT antigen and assayed for their capacity to proliferate and release LMF as just described.

\section{RESULTS}

Antigen-induced proliferation of human PBL in vitro. BPO-KLH induced proliferation $(\mathrm{SI}<2)$ in the PBL of six out of nine adult individuals studied (Fig. 1a). In contrast, BPO-HGG, the protein carrier KLH alone, and HGG all failed to induce proliferation of the lymphocytes from all nine adults (Fig. 1a). Furthermore, in a separate set of experiments, DNP-KLH conjugates failed to induce proliferation in the lymphocytes of three of the individuals who were capable of responding to BPO-KLH (Table I). As expected, TT provoked lymphocyte proliferation in the PBL of all nine donors (Fig. $1 a$ and Table I). It should be noted that lymphocytes from all six cord bloods failed to proliferate $(\mathrm{SI}<2)$ in response to antigens (BPOKLH or TT) in spite of the fact that they all responded to phytohemagglutinin to an extent equal to that of adult lymphocytes. The mean \pm SD counts per minute of 6 -d phytohemagglutinin-stimulated cultures was $19,769 \pm 4,389$ for cord blood lymphocytes and 21,170 $\pm 4,706$ for adult lymphocytes. However, the SI for cord blood lymphocytes $(11.9 \pm 4.7)$ as lower than for adult lymphocytes $(24.0 \pm 4.9)$ because the background for unstimulated cultures $(1,656 \pm 415 \mathrm{cpm})$ of cord blood lymphocytes was more than that obtained for unstimulated adult lymphocytes $(881 \pm 61)$. Because cord blood lymphocytes responded to mitogen phytohemagglutinin but not to antigen (BPO-KLH or TT), the above data suggest that the observed proliferation of adult PBL in response to BP(-KLH was antigenspecific.

Antigen-induced helper factor production. In the next series of experiments we examined antigen- 

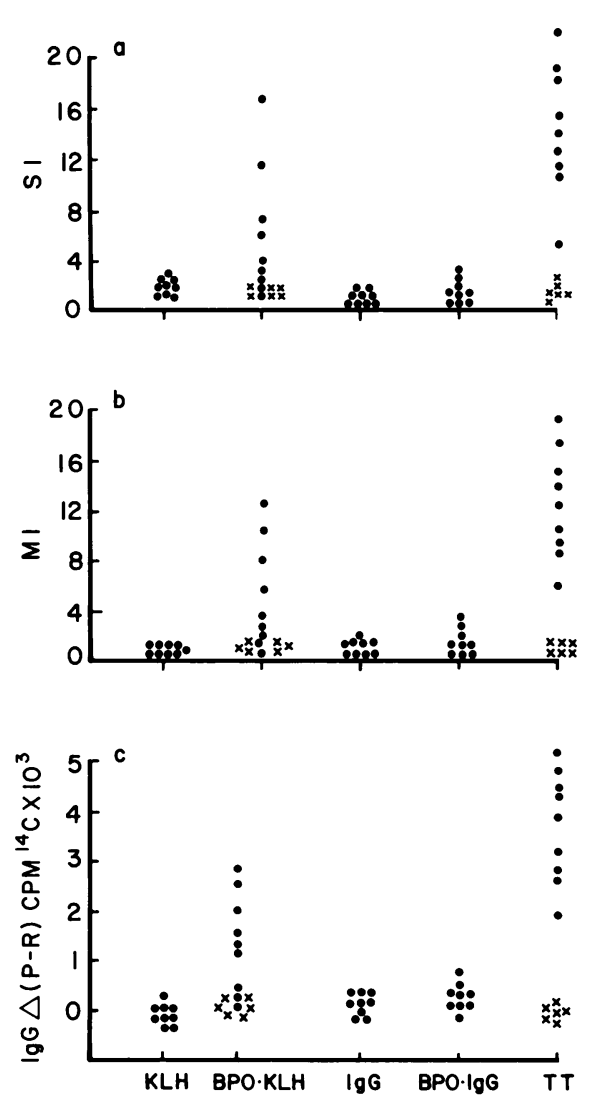

FIgure 1 Response of PBL from nine adult donors $(-)$ and six cord bloods $(\times)$ to BPO-KLH and TT. PBL responses to $\mathrm{KLH}$, IgG, and BPO-IgG are also presented. (a) Proliferation expressed as SI. (b) Release of the helper factor LMF into the supernates as assessed by ability to trigger proliferation of $B$ cells; results are expressed as MI. (c) Release of LMF as assessed by its ability to trigger de novo IgG synthesis in B cells. Results are expressed as the difference $(\Delta)$ of counts per minute of ${ }^{14} \mathrm{C}$ incorporated into IgG by cultures stimulated with paired $P$ and $R$ supernates as described in the text.

stimulated supernates for their content of the nonspecific helper factor LMF as assayed by their ability to induce proliferation and protein synthesis in allogeneic B cells. BPO-KLH induced LMF release by the lymphocytes of the six individuals who responded by cellular proliferation in vitro to BPO-KLH conjugates. Supernates from BPO-KLH-stimulated cultures of those six subjects triggered proliferation (MI $>2$, Fig. 1b) as well as IgG synthesis (Fig. 1c) in allogeneic B cells. In contrast, LMF was not released after incubation of the lymphocytes with BPO-HGG conjugates, KLH alone, or HGG. TT antigen caused LMF release from the lymphocytes of all nine adult individuals tested. In contrast, neither TT nor BPOKLH caused LMF release from cord blood lymphocytes (Fig. $1 b, c)$.

$B P O$-specific inhibition of lymphocyte proliferation by preincubation with BPO-HGG conjugates. The effect of in vitro incubation of lymphocytes with various conjugates on the proliferative response to antigen was assessed in the six individuals whose lymphocytes proliferated in response to BPO-KLH. Preincubation of lymphocytes with BPO-HGG resulted in significant inhibition of the proliferative response to the BPO-KLH conjugates but not to TT antigen (Fig. $2 a$ and Table I). In a total of nine experiments carried on the lymphocytes of these six individuals (Fig. $2 a$ and Table I), preincubation with BPO-HGG caused a mean inhibition of the BPO-KLH response of $68 \%$ and a mean inhibition of the TT response was $14 \%(P<0.01)$. Furthermore, in these nine experiments, the mean inhibition of the BPOKLH proliferative responses by preincubation with BPO-HGG $(68 \%)$ was also statistically significant $(P<0.01)$ when compared with the mean inhibition induced by preincubation with HGG alone (11\%). In contrast, preincubation of lymphocytes with BPO-HSA (Fig. $2 a$, six experiments) or DNP-HGG (Table I, three experiments) did not result in inhibition of the proliferative response to $\mathrm{BPO}-\mathrm{KLH}$. In the six experiments depicted on Fig. $2 a$, no inhibition of the BPO$\mathrm{KLH}$ response was seen with preincubation with BPO-HSA $(16 \%)$. In the three experiments shown in Table I, the mean inhibition of the BPO-KLH response induced by preincubation with DNP-HGG (21\%) was not significantly different than that seen in cultures preincubated with HGG (19\%) nor was it significantly different from the inhibition seen in cultures that had been preincubated with DNP-HGG and stimulated with TT $(15 \%)$. In these same three experiments, preincubation with BPO-HGG induced significant inhibition $(69 \%)$ of the BPO-KLH response when compared to preincubation with either HGG $(21 \%)$ or DNP-HGG $(19 \%) ; P$ values $<0.05$. Hence, the suppression of lymphocyte proliferation in response to BPO-KLH appears to be specific for BPO-HGG.

$B P O-$ specific inhibition of LMF release by preincubation with BPO-HGG conjugates. Preincubation of lymphocytes with BPO-HGG compared to preincubation with BPO-KLH resulted in significant inhibition of LMF release by lymphocytes after stimulation with BPO-KLH (Figs. $2 b, c$ ). Inhibition of LMF activity was observed in two different assays: first there was inhibition of B-cell proliferation (mean inhibition $61 \%, P<0.01$ ). Second, there was inhibition of de novo immunoglobulin synthesis by $\mathrm{B}$ cells (mean inhibition $79 \%, P<0.01$ ). Preincubation with BPO-HSA or unconjugated HGG failed to influence BPO-KLH-induced LMF release. Preincubation with BPO-HGG did not affect TT-induced LMF release.

\section{DISCUSSION}

These experiments were undertaken to examine whether hapten-specific unresponsiveness can be 
TABLE I

Specificity of the Proliferative Response to BPO-KLH and of the Inhibition of This Response by BPO-HCG

\begin{tabular}{|c|c|c|c|c|c|c|}
\hline \multirow[b]{2}{*}{ Donor } & \multirow{2}{*}{$\begin{array}{l}\text { Preincubation } \\
\text { with }\end{array}$} & \multirow[b]{2}{*}{-} & \multicolumn{4}{|c|}{ cpm $\left[{ }^{3} \mathrm{H}\right]$ thymidine incorporated/culture stimulated with } \\
\hline & & & BPO-HGG & KLH & DNP-KLH & TT \\
\hline \multirow[t]{4}{*}{1} & - & $330 \pm 68$ & $3,411 \pm 427$ & $508 \pm 101$ & $497 \pm 111$ & $6,792 \pm 663$ \\
\hline & HGG & $278 \pm 77$ & $2,722 \pm 340$ & $463 \pm 137$ & $343 \pm 108$ & $5,506 \pm 716$ \\
\hline & $\mathrm{BPO} / \mathrm{HGG}$ & $291 \pm 61$ & $1,353 \pm 181$ & $389 \pm 152$ & $428 \pm 203$ & $5,817 \pm 680$ \\
\hline & DNP-HGG & $303 \pm 54$ & $2,680 \pm 227$ & $446 \pm 164$ & $386 \pm 125$ & $6,012 \pm 824$ \\
\hline \multirow[t]{4}{*}{2} & - & $156 \pm 59$ & $3,082 \pm 526$ & $217 \pm 27$ & $203 \pm 28$ & $10,326 \pm 1,128$ \\
\hline & HGG & $138 \pm 42$ & $2,423 \pm 398$ & $256 \pm 91$ & $262 \pm 44$ & $8,687 \pm 854$ \\
\hline & BPO-HGG & $186 \pm 69$ & $1,052 \pm 208$ & $274 \pm 36$ & $359 \pm 47$ & $8,338 \pm 687$ \\
\hline & DNP-HGG & $105 \pm 35$ & $2,407 \pm 263$ & $192 \pm 48$ & $124 \pm 30$ & $9,107 \pm 596$ \\
\hline \multirow[t]{4}{*}{3} & - & $722 \pm 169$ & $8,985 \pm 1,154$ & - & $578 \pm 147$ & $22,107 \pm 1,433$ \\
\hline & HGG & $665 \pm 73$ & $7,325 \pm 907$ & - & $439 \pm 184$ & $19,729 \pm 862$ \\
\hline & BPO-HGG & $582 \pm 112$ & $2,409 \pm 563$ & - & $526 \pm 106$ & $18,772 \pm 1,649$ \\
\hline & DNP-HGG & $561 \pm 89$ & $7,138 \pm 328$ & - & $482 \pm 150$ & $18,438 \pm 1,297$ \\
\hline
\end{tabular}

Results represent mean $\pm \mathrm{SD}$ of triplicate cultures.

induced by HGG in PBL in vitro. BPO was chosen for three reassons: $(a)$ a large number of individuals receive penicillin. Both humoral and cell-mediated immunity to penicillin has been demonstrated in vivo and in vitro $(16-18) ;(b)$ it is well characterized $(8,9)$;
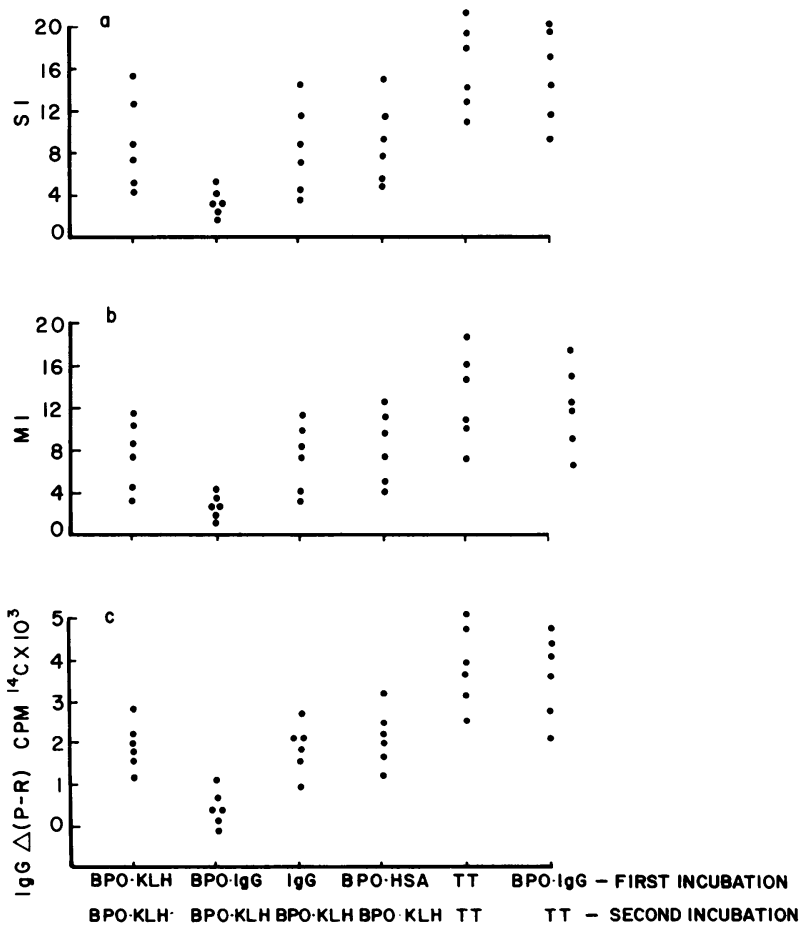

FIGURE 2 Effect of preincubation of PBL with different BPO-conjugates on the ability of these lymphocytes to proliferate $(a)$ and release the T-cell helper factor LMF ( $b$ and $c$ ). Preincubations were carried out as described in the text before culture. Frames $a, b$, and $c$ as described for Fig. 1 . and $(c)$ it is responsible for over half of the immediate hypersensitivity reactions to penicillin $(10,19,20)$. Initially, we determined whether conjugates of BPO to autologous or heterologous protein carriers elicit lymphocyte proliferation and the formation of nonspecific LMF in vitro. Lymphocytes from six of the nine subjects responded with significant proliferation and LMF production to BPO-KLH (Fig. 1). In contrast, none responded to BPO-HGG, HGG, KLH, or DNP$\mathrm{KLH}$ (Fig. $1 a$ and Table I). Because both lymphocyte proliferation and LMF production are T-cell functions (21), it is clear that in these responding individuals BPO-KLH is triggering the $\mathrm{T}$ cells. Several reasons could explain the failure of BPO-KLH to elicit significant $\mathrm{T}$-cell triggering in three out of nine individuals: $(a)$ a low level of cellular immunity to BPO in these subjects because of the length of time since they received penicillin; $(b)$ a failure of BPO-KLH to cross-react with similar antigenic determinants previously recognized by $\mathrm{T}$ cells: $(c)$ a low level of KLH-specific cells. Although others have recently reported that KLH elicits lymphocyte proliferation under different culture conditions in vitro (22), in our hands KLH alone did not cause a primary response in vitro. Furthermore, lymphocyte proliferation in response to BPO-KLH was not the result of nonspecific modification of the KLH molecule by the BPO haptenic groups because the conjugates of KLH to the DNP hapten did not induce $\mathrm{T}$-cell proliferation in three individuals studied and whose lymphocytes responded to BPO-KLH (Table I). Thus, in the six individuals who responded to $\mathrm{BPO}-\mathrm{KLH}$, this response was specific to the BPO-KLH conjugate.

Lymphocyte proliferation induced by BPO-KLH was not the result of polyclonal activation, because 
lymphocytes from the cord blood of newborns whose mothers did not receive penicillin during pregnancy failed to respond to BPO-KLH. In contrast, cord blood lymphocytes proliferated in response after exposure to the mitogen phytohemagglutinin. The cord blood response to phytohemagglutinin was comparable to that of adults when the results were expressed as counts per minute but was half the adult response when the results were expressed as SI. This is because the background for cord blood lymphocytes is known to be higher than for adult lymphocytes (23).

Preincubation of lymphocytes from all six subjects with BPO-HGG resulted in significant inhibition of the ability of the lymphocytes to proliferate and release $L M F$ in response to subsequent stimulation with BPO-KLH. This effect was BPO-specific because lymphocytes preincubated with BPO-HGG proliferated normally and released normal amounts of LMF on subsequent stimulation with the antigen TT. BPOHGG induction of unresponsiveness appears to be specific for the BPO-HGG conjugates. Indeed neither preincubation with unconjugated autologous HGG nor preincubation with conjugates of DNP with HGG resulted in the induction of lymphocyte unresponsiveness (Fig. 2, Table I), indicating that not all conjugates of HGG to haptens such as DNP are tolerogenic to $T$ cells. Furthermore, preincubation with conjugates of BPO with $\mathrm{KLH}$ or HSA did not result in the induction of lymphocyte unresponsiveness to BPO-KLH (Fig. 2), indicating that not all conjugates of BPO to self antigen such as HSA are necessarily tolerogenic.

Because lymphocyte proliferation and LFM release are both T-cell functions in man (21), it is clear that preincubation with BPO-HGG induced T-cell unresponsiveness. This suggests that helper $\mathrm{T}$ cells are made tolerant, confirming the observation made in mice that $\mathrm{T}$ cells as well as $\mathrm{B}$ cells are rendered tolerant by hapten isologous IgG (24). Nonetheless, how BPO-HGG made $T$ cells unresponsive is unclear. Extensive washings of the cells before stimulation with the immunogenic conjugate appears to rule out a simple physical blockade. But whether helper $\mathrm{T}$ cells were made unresponsive by receptor blockade or whether BPO-HGG could bind directly to and activate specific suppressor $\mathrm{T}$ cells which in man have a receptor for the $\mathrm{Fc}_{\mathrm{c}}$ portion of $\mathrm{IgG}$ is unknown (25). We favor the former i.e. helper T cells are made tolerant by receptor blockade because recent data obtained in adult thymectomized mice demonstrated that helper $\mathrm{T}$ cells are made tolerant directly by hapten isologous IgG without suppressor T cells (26). Because B-cell unresponsiveness could be explained solely by the absence of T-cell helper activity, it is unknown whether B cells are rendered tolerant. To resolve these issues, experiments similar to those reported in this paper must be performed using purified sub- populations of $\mathrm{T}$ and $\mathrm{B}$ cells. However, because in vitro B-cell response is T-dependent, it may be safe to predict that when the techniques are developed to measure human BPO-specific antibody response in vitro, this response will be suppressed by BPOHGG conjugates in humans as has already been shown in experimental animals (4).

Finally, the potential therapeutic importance of the induction of tolerance in man is worth mentioning. For example, hapten-specific tolerance induced by autologous human IgG might prevent allergic reactions caused by haptens, including penicillin hypersensitivity and reactions to the toxic gas trimellitic anhydride (27). Furthermore, and perhaps of greater clinical importance, several laboratories including our own are involved in the transformation of complete allergens such as ragweed $\operatorname{IgE}$ into tolerogen by linkage to nonimmunogenic carriers (28). The effectiveness of this approach has been very recently demonstrated by King in experimental animals (29). A similar approach in man might improve immunotherapy by selectively abrogating antibody to unwanted allergen.

\section{ACKNOWLEDGMENTS}

We wish to thank Drs. Fred S. Rosen and Harvey Colten for reviewing the manuscript and Ms. Lynne Kilham and Mr. Tom Brezina for technical assistance.

This work was supported by U. S. Public Health Service grant AI 05877, National Institute of Allergy and Infectious Diseases grant 5 R01 AI13867, American Cancer Society grant IM 178, grant funds from the Charles H. Hook Foundation, the National Foundation, March of Dimes, a Basil O'Connor research grant, and the $\mathrm{W}$. Rorer Corporation.

\section{REFERENCES}

1. Borel, Y. 1976. Isologous IgG-induced immunologic tolerance to haptens: a model of self versus non-self recognition. Transplant. Rev. 31: 3-22.

2. Katz, D. H., J. M. Davie, W. E. Paul, and B. Benacerraf. 1971. Carrier function in anti-hapten antibody responses. IV. Experimenta! conditions for the induction of haptenspecific tolerance or for the stimulation of anti-hapten anamnestic responses by 'nonimmunogenic' haptenpolypeptide conjugates. J. Exp. Med. 134: 201-223.

3. Lee, W. Y., and A. H. Sehon. 1978. Suppression of reaginic antibodies. Immunol. Rev. 41: 200-247.

4. Borel, Y., L. Kilham, N. Hyslop, and H. Borel. 1976. Isologous IgG induced tolerance to benzylpenicilloyl. Nature (Lond.). 261: 49-50.

5. Lee, W. Y., and A. H. Sehon. 1976. Suppression of reaginic antibody formation. IV. Suppression of reaginic antibodies to penicillin in the mouse. J. Immunol. 117: $927-934$.

6. Chiorazzi, N., Z. Eshhar, and D. H. Katz. 1976. Induction of immunological tolerance to the major determinant of penicillin: a therapeutic approach to penicillin allergy. Proc. Natl. Acad. Sci. U. S. A. 73: 2091-2095.

7. Otz, U., C. H. Schneider, A. L. deWeck, El Gruden, and T. J. Gill. 1978. Induction of immunological tolerance to the penicilloyl antigenic determinant. I. Evaluation 
of penicilloylated amino acid polyperms and copolymers in mice. Eur. J. Immunol. 8: 406-410.

8. Parker, C. W. 1965. Immunochemical mechanisms in penicillin allergy. Fed. Proc. 24: 51-54.

9. Levine, B. B., A. P. Redmond, M. J. Fellner, H. E. Voss, and V. Levytska. 1966. Penicillin allergy and the heterogeneous immune responses of man to benzylpenicillin. J. Clin. Invest. 45: 1895-1906.

10. Adkinson, N. F., W. L. Thompson, W. C. Maddrey, and L. Lichtenstein. 1971. Routine use of penicillin skin testing on an inpatient service. N. Engl. J. Med. 285: 22-24.

11. Hyslop, N. E., and R. M. Milligan. 1974. Chromatography of penicillin, penicilloates, and penicilloylamides on dextran gels. Antimicrob. Agents Chemother. 5: 617629.

12. Golan, D. T., and Y. Borel. 1971. Non-antigenicity and immunologic tolerance. The role of the carrier in the induction of tolerance to the hapten. J. Exp. Med. 134: 1046.

13. Boyum, A. 1968. Isolation of mononuclear cells and granulocytes from human blood. Scand. J. Clin. Lab. Invest. 21: 77-80.

14. Mudawwar, F., E. Yunis, and R. S. Geha. 1978. Antigenspecific helper factor in man.J. Exp. Med. 148: 1032-1046.

15. Geha, R. S., E. Schneeberger, F. S. Rosen, and E. Merler. 1973. Interaction of human thymus-derived ( $T$ ) and nonthymus-derived (B) lymphocytes in vitro. Induction of proliferation and antibody synthesis in B lymphocytes by a soluble factor released from antigen-stimulated T lymphocytes. J. Exp. Med. 138: 1230-1247.

16. Levine, B. B., M. J. Fellner, and V. Levytska. 1966. Benzylpenicilloyl specific serum antibodies to penicillin man. J. Immunol. 96: 707-726.

17. Klaus, M. V., and M. F. Fellner. 1973. Penicilloylspecific serum antibodies in man. J. Gerontol. 28: 312-316.

18. Fellner, M. J., and R. L. Baer. 1967. Response of lymphocytes to penicillin: comparison with skin tests and circulating antibodies in man. Nature (Lond.). 216: 803-804.
19. Levine, B. B., and D. M. Zolov. 1969. Prediction of penicillin allergy by immunological tests. J. Allergy. 43: 231-244.

20. Vickers, M. R., and A. S. K. Assem. 1974. Tests for penicillin allergy in man. I. Carrier effect on response to penicilloyl conjugates. J. Immunol. 26: 425-440.

21. Geha, R. S., and E. Merler. 1974. Human lymphocyte mitogenic factor: synthesis by sensitized thymus derived lymphocytes, dependence of expression on the presence of antigen. Cell. Immunol. 10: 86-104.

22. Hensen, E. J., and B. G. Elferink. 1979. Primary sensitization and restimulation of human lymphocytes with soluble antigen in vitro. Nature (Lond.). 277: 223-225.

23. Campbell, A. C., C. Waller, J. Wood, A. Aynsley-Green, and V. Yu. 1974. Lymphocyte subpopulations in the blood of newborn infants. Clin. Exp. Immunol. 18: 469-482.

24. Borel, Y., C. L. Reinisch, and S. F. Schlossman. 1975. $T$ and $B$ cell in hapten-specific carrier determined tolerance. J. Exp. Med. 142: 1254-1262.

25. Moretta, L., S. R. Webb, C. E. Grossi, P. M. Lydyard, and M. D. Cooper. 1977. Functional analysis of two human $\mathrm{T}$ cell subpopulations: help and suppression of B-cell responses by $\mathrm{T}$ cells bearing receptors for IgM or IgG. J. Exp. Med. 146: 184-200.

26. Borel, Y., L. Kilham, S. F. Kurtz, and C. L. Reinisch. 1980. Dichotomy between the induction of suppressor cells and immunologic tolerance by adult thymectomy. J. Exp. Med. In press.

27. Patterson, R., C. R. Zeiss, M. Roberts, J. J. Pruzansky, P. Wolkonsky, and R. Chacon. 1978. Human antihapten antibodies in trimellitic anhydride inhalation reactions. J. Clin. Invest. 62: 971-978.

28. Borel, Y., T. P. King, K. J. Sinclair, and K. J. Block. 1979. Suppression by antigen E-IgG conjugates of the IgE antibody response to ragweed IgE. J. Allergy Clin. Immunol. 63: 176-177. (Abstr.)

29. King, T. P., L. Kochoumian, and N. Chiorazzi. 1979. Immunological properties of conjugates of ragweed pollen antigen $\mathrm{E}$ wity methoxypolyethylene glycol or a copolymer of D-glutamic acid and D-lysine. J. Exp. Med. 149: 424-435. 